\title{
Is the use of triglyceride-glucose (TyG) index to recognize glucose disorders really practical?
}

\author{
Javad Alizargar ${ }^{1} \cdot$ Nan-Chen Hsieh ${ }^{2} \cdot$ Shu-Fang Vivienne $\mathrm{Wu}^{3}$ \\ Received: 2 March 2020 / Revised: 2 March 2020 / Accepted: 18 March 2020 / Published online: 15 May 2020 \\ (C) Springer-Verlag GmbH Germany, part of Springer Nature 2020
}

We read with great interest the article by Simental-Mendía et al. [1] that authors had shown triglyceride-glucose (TyG) index has a high sensitivity and specificity to detect impaired fasting glucose, impaired glucose tolerance, and also type 2 diabetes. However, in their article, certain points should have been considered and some possible errors should be rectified.

Simental-Mendía et al. [1] mentioned an article in 2008 [2] as the reference they used to calculate the TyG index. This reference used this following formula: $\mathrm{Ln}$ (fasting triglycerides $[\mathrm{mg} / \mathrm{dL}] \times$ fasting glucose $[\mathrm{mg} / \mathrm{dL}] / 2)$. Apparently, Simental-Mendía et al. [1] used this following slightly different formula: $\mathrm{Ln}$ (fasting triglycerides $[\mathrm{mg} / \mathrm{dL}] \times$ fasting glucose $[\mathrm{mg} / \mathrm{dL}]) / 2$, unlike their reference, possibly by mistake. So new calculations and new results might be expected by using the correct formula. Or if they just wrote a wrong formula in subheading "measurements" of their article or there is any reason to use a different formula, they should clarify.

Another key factor in using the TyG index is the real practical value of this index in the clinical practice. As the authors had previously stated [3], use of TyG index can be considered valuable, only if it adds to the current prognostic values of

Communicated by Peter de Winter

See related article, https://doi.org/10.1007/s00431-020-03644-1

Javad Alizargar

jaz.tmu@gmail.com

Nan-Chen Hsieh

nchsieh@ntunhs.edu.tw

Shu-Fang Vivienne Wu

shufang@ntunhs.edu.tw

1 Research Center for Healthcare Industry Innovation, National Taipei University of Nursing and Health Sciences, Taipei City 112, Taiwan

2 Department of Information Management, National Taipei University of Nursing and Health Sciences, Taipei City 112, Taiwan

3 College of Nursing, School of Nursing, National Taipei University of Nursing and Health Sciences, Taipei City 112, Taiwan fasting glucose and triglyceride values. If Simental-Mendía et al. [1] could provide different sets of statistical analysis in which they only use fasting glucose or triglyceride $\backslash$ levels with the same statistical settings they used in their article, and compare them with each other, readers and future investigators could see whether the use of TyG index is justifiable or not.

Authors' contributions JA wrote the first manuscript draft. SFVW and $\mathrm{NCH}$ helped in the literature review. SFVW contributed to manuscript revisions. All authors approved the final version.

\section{References}

1. Simental-Mendía LE, Gamboa-Gómez CI, Aradillas-García C, Rodríguez-Morán M, Guerrero-Romero F (2020) The triglyceride and glucose index is a useful biomarker to recognize glucose disorders in apparently healthy children and adolescents. Eur J Pediatr

2. Simental-Mendia LE, Rodriguez-Moran M, Guerrero-Romero F (2008) The product of fasting glucose and triglycerides as surrogate for identifying insulin resistance in apparently healthy subjects. Metab Syndr Relat Disord 6(4):299-304

3. Alizargar J, Bai C-H, Hsieh N-C, Wu S-FV (2020) Use of the triglyceride-glucose index (TyG) in cardiovascular disease patients. Cardiovasc Diabetol 19(1):8

Publisher's note Springer Nature remains neutral with regard to jurisdictional claims in published maps and institutional affiliations. 